\section{Structure and Dynamics of Single-isoform Recombinant Neuronal Human Tubulin ${ }^{*[5}$}

Received for publication, April 5, 2016, and in revised form, April 19, 2016 Published, JBC Papers in Press, April 25, 2016, DOI 10.1074/jbc.C116.731133

Annapurna Vemu ${ }^{\neq 1}$, Joseph Atherton ${ }^{\$ 1}$, Jeffrey O. Spector ${ }^{\neq 1}$, Agnieszka Szyk $^{\ddagger}$, Carolyn A. Moores ${ }^{\$ 2}$, and Antonina Roll-Mecak ${ }^{ \pm \uparrow 3}$ From the ${ }^{\ddagger}$ Cell Biology and Biophysics Unit, NINDS, and "Biophysics Center, NHLBI, National Institutes of Health, Bethesda, Maryland 20892 and the $\S$ Institute of Structural and Molecular Biology, Department of Biological Sciences, Birkbeck College, University of London,

London WC1E, United Kingdom

Microtubules are polymers that cycle stochastically between polymerization and depolymerization, i.e. they exhibit "dynamic instability." This behavior is crucial for cell division, motility, and differentiation. Although studies in the last decade have made fundamental breakthroughs in our understanding of how cellular effectors modulate microtubule dynamics, analysis of the relationship between tubulin sequence, structure, and dynamics has been held back by a lack of dynamics measurements with and structural characterization of homogeneous isotypically pure engineered tubulin. Here, we report for the first time the cryo-EM structure and in vitro dynamics parameters of recombinant isotypically pure human tubulin. $\alpha 1 \mathrm{~A} / \beta \mathrm{III}$ is a purely neuronal tubulin isoform. The $4.2-\AA$ structure of posttranslationally unmodified human $\alpha 1 \mathrm{~A} / \beta \mathrm{III}$ microtubules shows overall similarity to that of heterogeneous brain microtubules, but it is distinguished by subtle differences at polymerization interfaces, which are hot spots for sequence divergence between tubulin isoforms. In vitro dynamics assays show that, like mosaic brain microtubules, recombinant homogeneous microtubules undergo dynamic instability, but they polymerize slower and have fewer catastrophes. Interestingly, we find that epitaxial growth of $\alpha 1 \mathrm{~A} / \beta \mathrm{III}$ microtubules from heterogeneous brain seeds is inefficient but can be fully rescued by incorporating as little as $5 \%$ of brain tubulin into the homogeneous $\alpha 1 \mathrm{~A} /$ $\beta$ III lattice. Our study establishes a system to examine the struc-

* This work was supported by grants from the Medical Research Council, United Kingdom (to J. A. and C. A. M.), and by the Intramural Programs of the NINDS and the NHLBI, National Institutes of Health (to A. V., J. O. S., A. S. and A. R.-M.). The content is solely the responsibility of the authors and does not necessarily represent the official views of the National Institutes of Health.

- This article was selected as a Paper of the Week.

¿Author's Choice-Final version free via Creative Commons CC-BY license.

5 This article contains supplemental Table 1 and supplemental Movies 1-3.

The atomic coordinates and structure factors (code 5JCO) have been deposited in the Protein Data Bank (http://wwpdb.org/).

The EMDB accession code for the GMPCPP $\alpha 1 A / \beta I I I$ microtubule reconstruction is 8150.

${ }^{1}$ These authors contributed equally to this work.

${ }^{2}$ To whom correspondence may be addressed: Institute of Structural and Molecular Biology, Birkbeck College, London WC1E 7HX, United Kingdom. Tel.: 020-7631-6858; E-mail: c.moores@mail.cryst.bbk.ac.uk.

${ }^{3}$ To whom correspondence may be addressed: Cell Biology and Biophysics Unit, Porter Neuroscience Research Center, National Institutes of Health, Bldg. 35, Rm. 3B-203, 35 Convent Dr., MSC 3700, Bethesda, MD 20892-3700. Tel.: 301-814-8119; E-mail: Antonina@mail.nih.gov. ture and dynamics of mammalian microtubules with well defined tubulin species and is a first and necessary step toward uncovering how tubulin genetic and chemical diversity is exploited to modulate intrinsic microtubule dynamics.

Microtubules cycle stochastically between periods of polymerization and depolymerization, i.e. they exhibit "dynamic instability" (1). This behavior is crucial in cell division, motility, and differentiation. Despite the discovery of dynamic instability more than 30 years ago (1) and fundamental breakthroughs in our understanding of microtubule dynamics modulation by cellular effectors $(2,3)$, analysis of the relationship between tubulin sequence, structure, and dynamics has been held back by a lack of structural and in vitro dynamics data with homogeneous isotypically pure engineered tubulin. Eukaryotes have multiple tubulin genes (humans have eight $\alpha$ - and eight $\beta$-tubulin isotypes) with tissue-specific distributions (4). Some microtubules are isotype mixtures, and others are formed from a predominant single isotype (5). Moreover, tubulin is subject to abundant and chemically diverse post-translational modifications that include acetylation, detyrosination, phosphorylation, glutamylation, glycylation, and amination $(6,7)$. Virtually all biochemical studies have used tubulin purified from mammalian brain tissue through multiple cycles of in vitro depolymerization and polymerization (8). Although tubulin is abundant in this source, the resulting material is highly heterogeneous, being composed of multiple tubulin isotypes bearing chemically diverse and abundant posttranslational modifications (9-11). More than 22 different charge variants are repolymerized in random fashion for in vitro polymerization assays (12). Thus, microtubules used for in vitro dynamics assays have been mosaic, with random distributions of isoforms and post-translational modifications. Moreover, this purification procedure selects tubulin subpopulations that polymerize robustly while discarding those that do not. Efforts to reduce metazoan tubulin heterogeneity exploited differences in isoform compositions between various tissues or cell lines (e.g. avian erythrocytes (13) and HeLa cells (14)) or the use of isoform-specific antibodies for immunopurification (15). However, neither of these approaches yielded homogeneous single-isoform tubulin. Here, we report for the first time the expression and purification of recombinant isotypically pure unmodified human tubulin competent for in vitro dynamics assays and report its dynamic parameters as well as cryo-EM structure at 4.2 A resolution. We find that isotypically pure unmodified $\alpha 1 \mathrm{~A} / \beta \mathrm{III}-$ tubulin exhibits subtle differences in dynamics when compared with heterogeneous brain tubulin, consistent with the small conformational rearrangements at tubulin polymerization interfaces revealed by our near-atomic resolution structure of $\alpha 1 \mathrm{~A} / \beta$ III microtubules. Our study establishes a system to examine the structure and dynamics of mammalian microtubules with well defined $\alpha$ and $\beta$-tubulin species and is a first and necessary step toward exploring the biophysical correlates 
between sequence, structure, and dynamics for mammalian microtubules.

\section{Experimental Procedures}

Expression and Purification of Human Recombinant Tubulin Constructs-Codon-optimized genes for human $\alpha 1 \mathrm{~A}$-tubulin (NP_001257328) with an internal His tag in the acetylation loop and a PreScission protease-cleavable C-terminally FLAGtagged $\beta$ III-tubulin (NM_006077) were custom-synthesized by Integrated DNA Technologies and cloned into a pFastBac ${ }^{\mathrm{TM}}$ Dual vector as described previously $(16,17)$. The internal His tag in $\alpha$-tubulin allowed production of an $\alpha$-tubulin ending in its natural C-terminal tyrosine $(17,18)$. Without an affinitybased selection for $\alpha$-tubulin, the final sample contains $\sim 30 \%$ contamination with endogenous insect $\alpha$-tubulin species that can be variable from construct to construct. The Bac-to-Bac System (Life Technologies, Inc.) was used to generate bacmids for baculovirus protein expression. High-Five or SF9 cells were grown to a density between 1.3 and $1.6 \times 10^{6}$ cells $/ \mathrm{ml}$ and infected with viruses at the multiplicity of infection of 1 . Cultures were grown in suspension for $48 \mathrm{~h}$, and cell pellets were collected, washed in PBS, and flash-frozen. Cells were lysed by gentle sonication in $1 \times$ BRB80 buffer (80 mM PIPES, pH 6.9, 1 $\mathrm{mm} \mathrm{MgCl}_{2}, 1 \mathrm{~mm}$ EGTA) with addition of: $0.5 \mathrm{~mm}$ ATP, $0.5 \mathrm{~mm}$ GTP, $1 \mathrm{mM}$ PMSF, and 25 units/ $\mu$ l benzonase nuclease. The lysate was supplemented with $500 \mathrm{~mm} \mathrm{KCl}$ and cleared by centrifugation $(15 \mathrm{~min}$ at $400,000 \times g)$. The crude supernatant (supplemented with $25 \mathrm{~mm}$ imidazole, $\mathrm{pH}$ 8.0) was loaded on a nickel-nitrilotriacetic acid column (Qiagen) equilibrated with high salt buffer (BRB80, $500 \mathrm{mM} \mathrm{KCl,} 25 \mathrm{~mm}$ imidazole). Histagged tubulin was eluted with $250 \mathrm{~mm}$ imidazole in BRB80 buffer. The eluate was further purified on anti-FLAG G1 affinity resin (Gen Script). FLAG-tagged tubulin was eluted by incubation with FLAG peptide (GenScript) at $0.25 \mathrm{~g} /$ liter concentration followed by removal of the tag by PreScission protease. A final purification step was performed on a Resource $Q$ anion exchange column (GE Healthcare) with a linear gradient from $100 \mathrm{~mm}$ to $1 \mathrm{M} \mathrm{KCl}$ in BRB80 buffer. Peak fractions were pooled and buffer-exchanged on a PD10 desalting column (GE Healthcare) equilibrated with BRB80, $20 \mu \mathrm{M}$ GTP. Small aliquots of tubulin were frozen in liquid nitrogen and stored at $-80^{\circ} \mathrm{C}$ until use. The purified tubulin was subjected to ESI-TOF LC-MS analysis and detected no endogenous tubulin or posttranslational modifications (Fig. 1A). The sensitivity of our mass spectrometric analyses is high enough to detect as little as $1 \%$ contaminating post-translationally modified tubulin species (17). The final yield is $\sim 1 \mathrm{mg}$ of $>99 \%$ recombinant isotypically pure $\alpha \beta$-tubulin per liter of SF9 cells.

Cryo-EM Sample Preparation and Data Collection-Recombinant human $\alpha 1 \mathrm{~A} / \beta \mathrm{III}$-tubulin was polymerized at a final concentration of $2.5 \mathrm{mg} / \mathrm{ml}$ in BRB80 buffer ( $80 \mathrm{~mm}$ PIPES, $2 \mathrm{~mm}$ $\mathrm{MgCl}_{2}, 1 \mathrm{~mm}$ EGTA, $1 \mathrm{~mm}$ DTT) with $1 \mathrm{~mm} \mathrm{GMPCPP}{ }^{4}$ or $2 \mathrm{~mm}$ GTP at $37^{\circ} \mathrm{C}$ for $1 \mathrm{~h}$. GMPCPP-bound microtubules were double-cycled by depolymerizing on ice and then repolymerized at

\footnotetext{
${ }^{4}$ The abbreviations used are: GMPCPP, guanylyl- $(\alpha, \beta)$-methylene-diphosphonate; AMPPNP, $5^{\prime}$-adenylyl- $\beta, \gamma$-imidodiphosphate; PDB, Protein Data Bank.
}

$37^{\circ} \mathrm{C}$ for $1 \mathrm{~h}$ with an additional $2 \mathrm{~mm}$ GMPCPP. Stabilized $\alpha 1 \mathrm{~A} / \beta$ III microtubules were diluted in BRB20 (20 mM PIPES, 2 $\mathrm{mm} \mathrm{MgCl}$, 1 mM EGTA, 1 mM DTT) to a final concentration of $2.5 \mu \mathrm{M}$. Human kinesin-3 motor domain (Kif1A, residues 1-361) (19) was diluted to $20 \mu \mathrm{M}$ in BRB20 with $2 \mathrm{mM}$ AMPPNP. The microtubules and motor were applied sequentially to glow-discharged $\mathrm{C}$-flat ${ }^{\mathrm{TM}}$ holey carbon grids (Protochips), and the sample was vitrified using a Vitrobot (FEI Co.). The presence of the kinesin motor domain allowed differentiation between $\alpha$ - and $\beta$-tubulin during processing. Images were collected with a DE20 direct electron detector (Direct Electron) on a FEI Tecnai G2 Polara operating at $300 \mathrm{kV}$ with a calibrated magnification of $\times 52,117$ corresponding to a final sampling of $1.22 \AA$ /pixel. A total electron dose of $\sim 50 \mathrm{e}^{-} / \AA^{2}$ over a 1.5 -s exposure and a frame rate of 15 frames/s was used, giving a total of 23 frames at $\sim 2.2$ electrons/frame. Dynamic microtubules grown from GMPCPP seeds were polymerized at $2 \mathrm{mg} / \mathrm{ml}$ for $30 \mathrm{~min}$, kept at $37^{\circ} \mathrm{C}$ throughout, and vitrified as above. Images were collected on a FEI Tecnai T12 operating at $120 \mathrm{kV}$ using a $4096 \times 4096$-pixel CCD camera (Gatan Inc.).

Data Processing for Three-dimensional ReconstructionIndividual $\sim 2.2 \mathrm{e}^{-} / \AA^{2}$ frames were globally aligned using IMOD scripts (20) then locally aligned using the Optical Flow approach (21) implemented in Xmipp (22). The full dose of $\sim 50$ $\mathrm{e}^{-} / \AA^{2}$ was used for particle picking and CTF determination in CTFFind3 (23), whereas $\sim 25 \mathrm{e}^{-} / \AA^{2}$ was used in particle processing to center particles and determine their Euler angles. Euler angles and shifts determined using $\sim 25 \mathrm{e}^{-} / \AA^{2}$ dose were used to generate reconstructions from either the first $\sim 25$ or $\sim 12 \mathrm{e}^{-} / \AA^{2}$ of the exposure. Kinesin-3 microtubules were manually boxed in Eman Boxer (24), serving as input for a set of custom-designed semi-automated single-particle processing scripts utilizing Spider and Frealign as described previously (25) with minor modifications. 10,164 particles or 142,296 asymmetric units were used in the final reconstruction, which was assessed for overfitting using a high resolution noise-substitution test (26). Using local resolution estimates determined with the blocres program in Bsoft, the reconstruction was sharpened with a $B$ factor of -180 up to a resolution of 5.5 or $4 \AA$ for visualization of kinesin or tubulin densities, respectively. The overall resolution of the reconstruction is $4.2 \AA$ (FSCtrue, 0.143 criteria) (26) encompassing a resolution range of $\sim 3.5-5.5 \AA$. The best regions of the reconstruction are within the tubulin portion of the complex (Figs. $1 B$ and 2 ) from which we built an $\alpha 1 \mathrm{~A} / \beta \mathrm{III}$ microtubule model. The quality of our reconstruction was sufficient to confirm that GMPCPP was found in the E-site (Fig. 1C) and GTP in the N-site.

Model Building and Refinement-The polypeptide model of the unmodified $\alpha 1 \mathrm{~A} / \beta \mathrm{III}$-tubulin GMPCPP microtubule was built directly into density in Coot (27) using PDB 3JAT (28) as a starting model. The structure was refined under symmetry restraints in REFMAC version 5.8 (29). Secondary structure and reference restraints based on the high resolution tubulin crystal structure PDB 4DRX (30) were generated with ProSMART (31). Model building in Coot and refinement in REFMAC were repeated iteratively until the quality of the model and fit were optimized (supplemental Table 1). 
In Vitro Microtubule Dynamics Assays-GMPCPP stabilized seeds were prepared as described (32). The GMPCPP seeds were immobilized in flow chambers using neutravidin as described previously (33). The final imaging buffer contained $1 \times$ BRB80 supplemented with $1 \mathrm{~mm}$ GTP, $100 \mathrm{~mm} \mathrm{KCl,} 1 \%$ pluronic F-127, and oxygen scavengers prepared as described (34). An objective heater (Bioptechs) was used to warm the chamber to $30^{\circ} \mathrm{C}$. All chambers were sealed and allowed to equilibrate on the microscope stage for $5 \mathrm{~min}$ prior to imaging. Dark field images were acquired every $5 \mathrm{~s}$ for $30 \mathrm{~min}$. For depolymerization rate measurements, the frame rate used was 40 frames/s. Imaging was performed on a Nikon Eclipse Ti-E equipped with a high NA dark field condenser, a $\times 100$ adjustable iris objective and a Hamamatsu Flash 4.0 version 2 camera with $2 \times 2$ binning. The final pixel size was $108 \mathrm{~nm}$. Dark field illumination was provided by a Lumencor SOLA SE-II light engine. A Nikon GIF filter was used to protect the seeds from excessive photodamage.

Dynamic Parameter Measurements-Using ImageJ, kymographs were generated from dark field images. Kymographs were traced by hand, and dynamic parameters were calculated. Growth and depolymerization rates were determined from the slope of the growing or depolymerizing microtubule in the kymographs. Catastrophe frequency was determined as the number of observed catastrophes divided by the total time spent in the growth phase. Extremely rare rescue events were observed under our experimental conditions and thus were not quantified. Mean microtubule lifetime was calculated as the average time a microtubule spent in the growth phase before a catastrophe. Mean microtubule length was calculated as the average length a microtubule reached before a catastrophe. The probability of nucleation was determined by determining the percentage of seeds that nucleated in $30 \mathrm{~min}$ in a field of view. Dynamicity was determined as defined in Toso etal. (35) as the sum of total growth and shortening lengths divided by total time.

\section{Results}

Near Atomic Resolution Structure of Single-isoform Human $\alpha 1 A / \beta I I I$ Microtubules-We selected for our study $\alpha 1 \mathrm{~A} / \beta \mathrm{III}-$ tubulin. $\beta$ III is a neuronal isoform that constitutes $25 \%$ of purified brain tubulin (10). It is expressed in non-neuronal tissues only during tumorigenesis $(36,37)$. It is also the most divergent of all $\beta$-tubulin isotypes. It is highly overexpressed in nonneuronal cells upon transformation and has been identified as a strong prognosticator of poor clinical outcomes (37). We expressed human $\alpha 1 \mathrm{~A} / \beta \mathrm{III}-$ tubulin in insect cells (16). Through a new double-selection strategy using affinity tags on both $\alpha$ - and $\beta$-tubulin, we produced $>99 \%$ homogeneous, modification-free, single-isotype human $\alpha \beta$-tubulin, free of contamination from endogenous insect tubulins (Fig. $1 A$ and see under "Experimental Procedures") that is assembly-competent in the absence of stabilizing drugs like taxol and thus suitable for in vitro dynamics assays. Our tagging scheme generates an $\alpha$-tubulin with a native $C$ terminus and thus this recombinant tubulin is suitable for the investigation of the effects of the tubulin detyrosination/tyrosination cycle on intrinsic microtubule dynamics and those mediated by the modification-dependent recruitment of cellular effectors $(38,39)$.
To gain insight into the assembly properties of $\alpha 1 \mathrm{~A} / \beta \mathrm{III}$ recombinant tubulin, we determined the structure of $\alpha 1 \mathrm{~A} / \beta \mathrm{III}$ microtubules in complex with the GTP analog GMPCPP at near-atomic resolution using cryo-electron microscopy and single-particle image reconstruction (Figs. $1 B$ and 2) (25). There is a resolution gradient in the reconstruction, with the best resolution $(\sim 3.5 \AA)$ within the body of the microtubule (encompassing a resolution range of $\sim 3.5-4.5 \AA$, Fig. $2 A$ ). The resolution range of the kinesin motor domain, used to facilitate reconstruction, is $\sim 4.5-5.5 \AA$. Overall, the reconstruction has a resolution of $4.2 \AA$ (Fourier shell correlation, 0.143 criterion (26), encompassing a resolution range of $\sim 3.5-5.5 \AA$ ) (Fig. $2, B$ and $C$ ). The reconstruction shows clearly resolved $\beta$-sheets and $\alpha$-helical pitch (Fig. 2, $D-F$ ). The majority (92\%) of human $\alpha 1 \mathrm{~A} / \beta \mathrm{III}$ GMPCPP microtubules have 14 protofilaments, similar to brain GMPCPP microtubules (40). The tubulin monomer consists of a well folded globular core and highly negatively charged and flexible C-terminal tails (41). The C-terminal tails are the locus of the greatest chemical heterogeneity in tubulin. They appear disordered in all microtubule structures to date either because (i) they have no unique well defined conformation or (ii) defined conformations unique to particular isoforms or post-translationally modified forms are lost during the iterative averaging used in EM reconstructions due to the high heterogeneity of these tails in brain tubulin samples. Despite the chemical homogeneity of our sample, there is no density attributable to them, indicating that they are intrinsically disordered unless engaged by an effector as seen for the tubulin tyrosine ligase like 7 glutamylase or the NDC80 complex (42-44).

Consistent with the high sequence conservation of the tubulin body, our structure is similar to that of heterogeneous mosaic mammalian brain GMPCPP microtubules, and the overall conformation of the tubulin dimers in our reconstruction is consistent with a GTP-like extended conformation (Fig. 1C) (28). The backbone root mean square deviation of our tubulin dimer model overlaid on that of the recently published structure of mammalian heterogeneous brain GMPCPP 14 protofilament microtubules is $<2 \AA$. A difference in the tubulin repeat distance is observed between $\alpha 1 \mathrm{~A} / \beta \mathrm{III}$ and brain microtubules as follows: $82.7 \pm 0.2$ versus $83.1 \pm 0.0 \AA$ measured from the EM reconstruction (i.e. model-independent); 82.6 versus $83.2 \AA$ measured by comparing models, for $\alpha 1 \mathrm{~A} / \beta \mathrm{III}$ and brain microtubules, respectively $(28,45)$. However, the tubulin repeat distance for the recombinant $\alpha 1 \mathrm{~A} / \beta \mathrm{III}$ microtubules $(\sim 82.7 \AA)$ is roughly comparable with the repeat distance for heterogeneous brain GMPCPP microtubules $(\sim 83 \AA)$, which is more extended than that of the GDP state $(\sim 81.5 \AA)(28,45)$. Nevertheless, we find two subtle differences that have the potential to impact polymerization dynamics. First, the loop connecting helices $\mathrm{H} 1$ and $\mathrm{H}^{\prime}{ }^{\prime}$ in $\beta$-tubulin shifts $\sim 3 \AA$ away from the H1'-S2 loop, which makes lateral contacts with the M-loop (microtubule loop) of the neighboring dimer (Fig. 1, $D$ and $E$ ). The M-loop is a sequence element crucial to lateral contacts between adjacent protofilaments. Strikingly, the H1'S2, H2-S3, and M-loops are a hot spot of sequence variation across $\beta$-tubulin isoforms (Fig. $1 F$ ), consistent with the structural plasticity we observe at this interface. Second, when one $\alpha$-protomer each of brain GMPCPP and recombinant $\alpha 1 \mathrm{~A} / \beta \mathrm{III}$ 
REPORT: Structure and Dynamics of Human $\alpha 1 A / \beta / I-$ tubulin
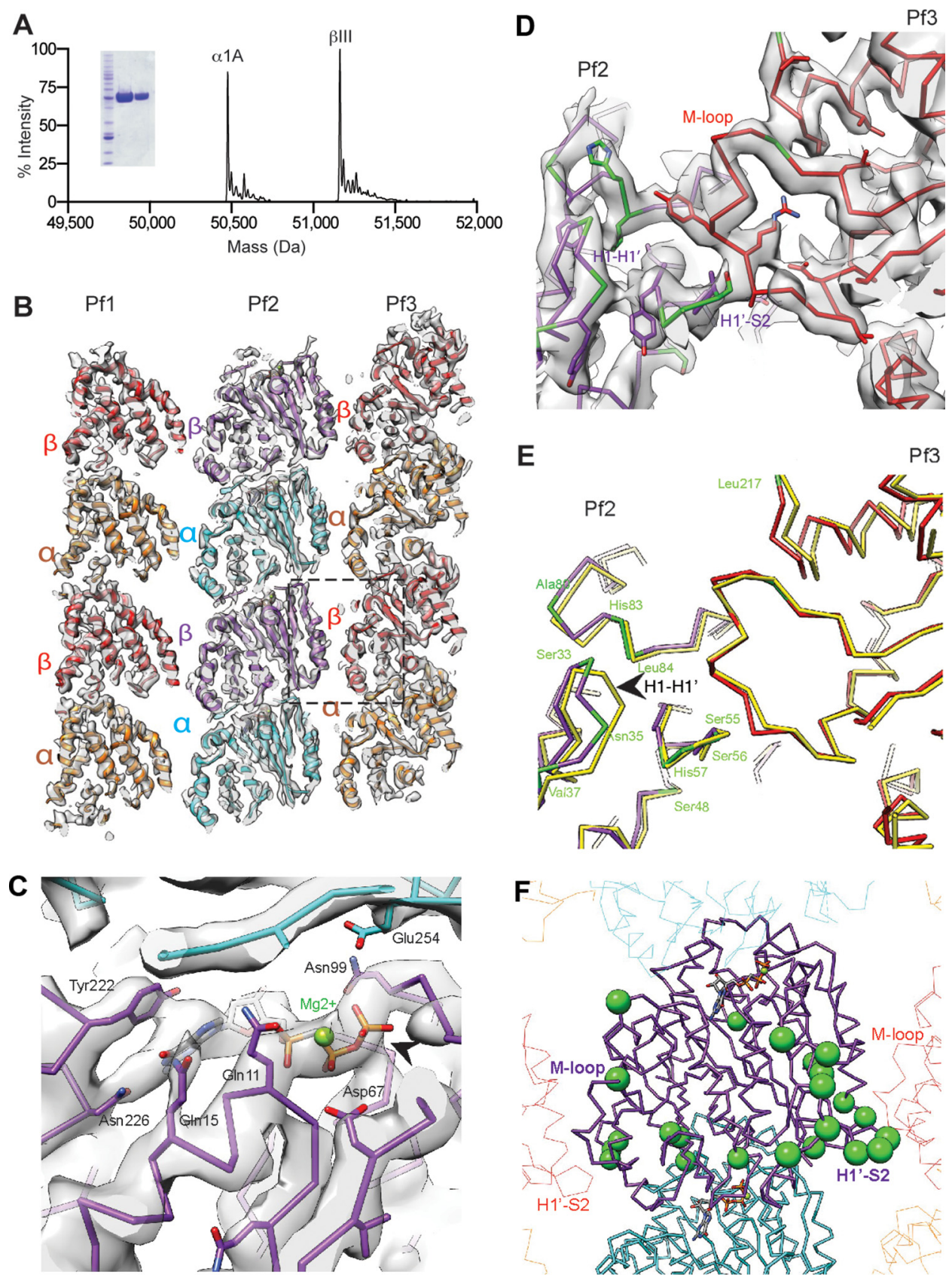

FIGURE 1. Structure of unmodified single-isoform human $\boldsymbol{\alpha} \mathbf{1 A} / \boldsymbol{\beta}$ III microtubules. $A$, mass spectra and SDS-polyacrylamide gel (inset) of recombinant human $\alpha 1 \mathrm{~A} / \beta \mathrm{III}$-tubulin purified to $>99 \%$ homogeneity. The experimentally determined masses for $\alpha 1 \mathrm{~A}$ - and $\beta$ III-tubulin were $50,477.8$ and $51,163.6 \mathrm{Da}$, respectively. The theoretical masses for $\alpha 1 \mathrm{~A}$ - and $\beta$ III-tubulin are 50,476.8 and 51,162.4 Da, respectively. $B$, cryo-EM map (4.2 $\AA$ resolution, $2.8 \sigma$ contour) and model of GMPCPP recombinant human $\alpha 1 \mathrm{~A} / \beta \mathrm{III}$ microtubules viewed from the lumen (three protofilaments shown). A central protofilament (Pf2) makes lateral contacts with adjacent protofilaments (Pf1 and Pf3); $\alpha$-tubulin, orange, $\beta$-tubulin, red (Pf1, Pf3); $\alpha$-tubulin, cyan; $\beta$-tubulin, purple (Pf2). C, E-site in $\beta$ III-tubulin shows clear density for GMPCPP and its three phosphate groups. D, model and map of the $\beta$ Ill-tubulin lateral interface (boxed and colored as in $B$ ). $\beta$ III-specific residues are in green. E, superposition of the $\alpha 1 \mathrm{~A} / \beta \mathrm{III}$ (colored as in $B$ ) and brain (PDB, 3JAT; atomistic models of brain microtubules use the $\beta$ ) isotype sequence because it constitutes $\sim 50 \%$ of these preparations $(28,44)$; yellow) microtubule structures; residues specific to $\beta$ III are in green. $F$, $\beta$ III sequence variability concentrates at the lateral interface. Green spheres denote residues that are different between the $\beta I I I$ and $\beta \| l$ isotypes, the most abundant tubulin isoforms in brain tubulin preparations (10).

GMPCPP microtubule protofilaments are superimposed, a clear displacement of successive recombinant $\alpha 1 \mathrm{~A} / \beta \mathrm{III}$ dimers becomes apparent (Fig. $3 A$ ). This propagates from the exchangeable GTP-site (E-site) and $\beta$ III-tubulin longitudinal interface and results in a progressive stagger that increases with each dimer along the protofilament, such that the first neighboring dimer is offset by $1.7 \AA$ (all C $\alpha$ root mean square deviation), the second by $3.4 \AA$, and so on. Together, these rel- atively subtle structural differences could contribute to differences in dynamic properties. Interestingly, we find that at $6 \mu \mathrm{M}$ $\alpha 1 \mathrm{~A} / \beta$ III-tubulin, $92 \%$ of $\alpha 1 \mathrm{~A} / \beta \mathrm{III}$ GMPCPP seeds nucleate microtubules but only $33 \%$ brain seeds nucleate $\alpha 1 \mathrm{~A} / \beta \mathrm{III}$ microtubules (Fig. $3 B$ ), suggestive of lattice mismatch effects between the brain microtubule seed and the lattice parameters of the growing $\alpha 1 \mathrm{~A} / \beta \mathrm{III}$ microtubule. This is consistent with the subtle structural differences between $\alpha 1 \mathrm{~A} / \beta \mathrm{III}$ and 


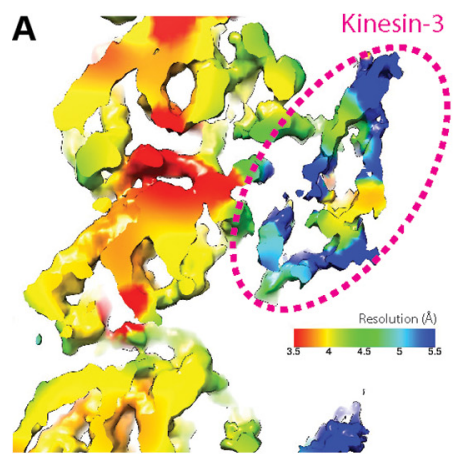

B
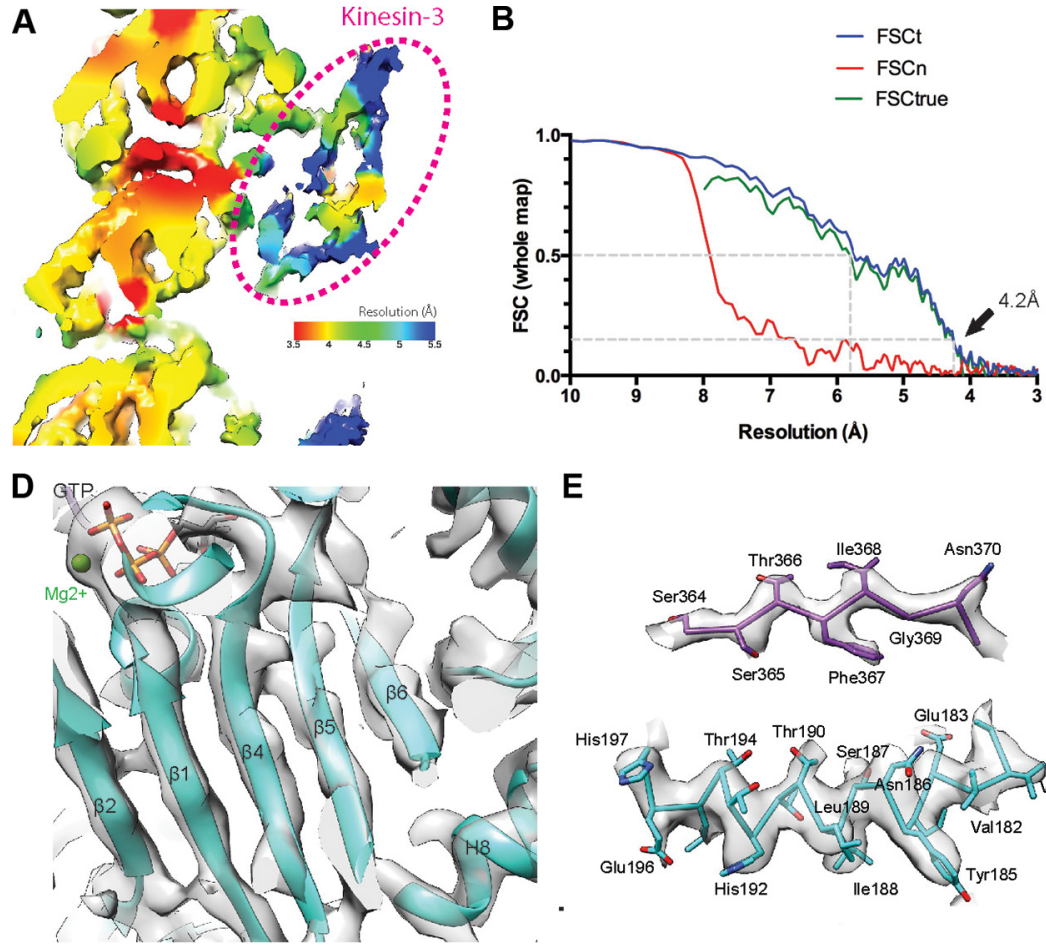

E
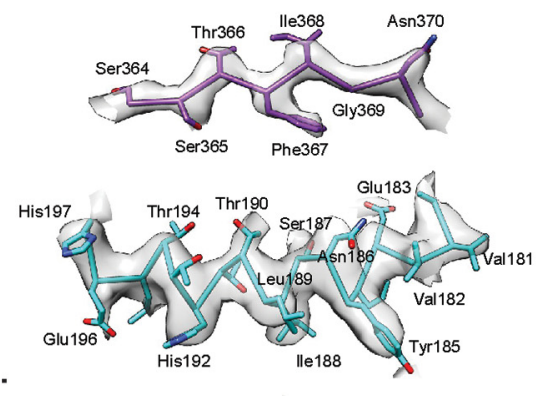

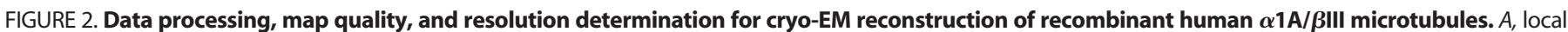

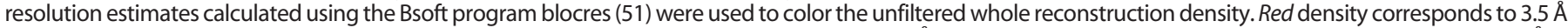

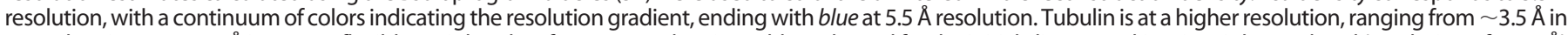

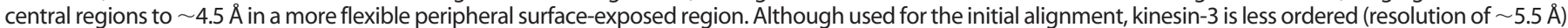

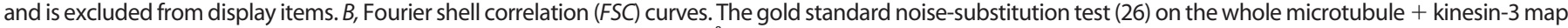

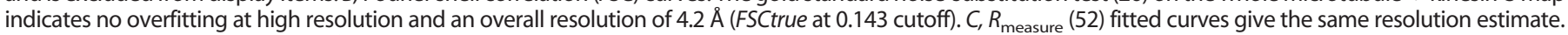

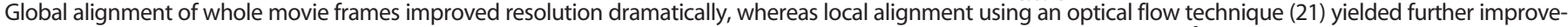

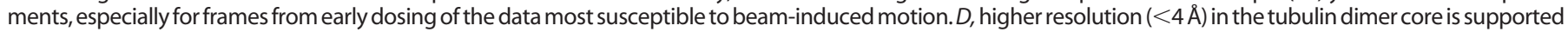

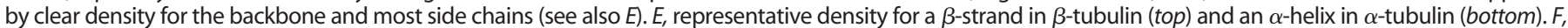

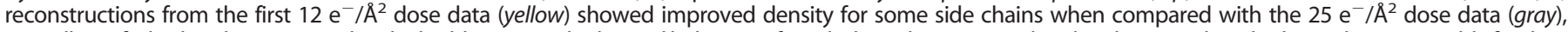

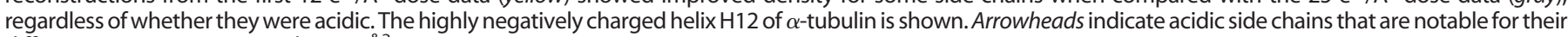
different appearance in 12 and $25 \mathrm{e}^{-} / \AA^{2}$ maps.

heterogeneous brain microtubules that we identified (Figs. 1, $D$ and $E$, and $3 A$ ). Unexpectedly, robust growth off brain seeds at $6 \mu \mathrm{M} \alpha 1 \mathrm{~A} / \beta \mathrm{III}$ could be rescued (from 33 to $91 \%$ ) if as little as $5 \%$ brain tubulin was added (Fig. $3 B$ ). Thus, a small level of tubulin heterogeneity can alleviate the nucleation defect that arises from the potential mismatch between the lattices of the two microtubule types. Our finding has intriguing consequences for the nucleation in vivo of microtubules composed of mixtures of tubulin isoforms.

In Vitro Dynamics of Single-isoform $\alpha 1 A / \beta I I I-t u b u l i n-T o$ determine dynamic parameters of single-isoform $\alpha 1 \mathrm{~A} / \beta \mathrm{III}-\mathrm{tu}$ bulin, we performed label-free in vitro dynamic assays using dark field microscopy (Fig. 4 and supplemental Movies 1 and 2) (46) so that our dynamic parameters are not confounded by effects arising from the addition of fluorescently labeled brain tubulin to the otherwise homogeneous microtubules. The $\alpha 1 \mathrm{~A} /$ $\beta I I I$ microtubules have the typical end appearance observed for brain microtubules consisting of a mixture of short sheet-like and blunter structures (Fig. 4B) (47). To quantify their dynamics, we generated kymographs from time-lapse imaging of dynamic microtubule assays (Fig. $4 \mathrm{C}$ ). The growth rates at the plus-end are $35 \%$ slower when compared with those of heterogeneous brain tubulin, whereas the minus-end growth rates are statistically indistinguishable. Consistent with this, the on-rate of $\alpha 1 \mathrm{~A} / \beta \mathrm{III}-$ tubulin at the plus-end is 1.8 dimers $\mathrm{s}^{-1} \mu \mathrm{M}^{-1}$ compared with the 3.6 dimers s ${ }^{-1} \mu \mathrm{M}^{-1}$ for brain tubulin (our measurements for brain microtubules are similar to those reported in Ref. 48). Dark field imaging allows data collection at the high frame rates needed to determine microtubule depolymerization rates with high accuracy ("Experimental Procedures" and see supplemental Movie 3). These measurements revealed that $\alpha 1 \mathrm{~A} / \beta \mathrm{III}$ microtubules depolymerize slower than brain microtubules $(30.5 \pm 1.3 \mu \mathrm{m} / \mathrm{min}$ versus $39.9 \pm 1.5$ $\mu \mathrm{m} / \mathrm{min}$; Fig. $4 D$ ). This suggests that microtubules with different chemical compositions (isoform or post-translational modifications) have the potential to generate different end depolymerization forces that could be harnessed to move cargo in the cell, such as chromosomes during cell division (49).

The catastrophe (the transition between growth and shrinkage) frequency of recombinant microtubules is slightly reduced by 20 and $44 \%$ at the plus- and minus-ends, respectively, when compared with heterogeneous brain tubulin (Fig. 4, $E$ and $F$ ). Interestingly, although $46 \%$ of brain microtubule exhibit growth at their minus ends, fewer than $7 \%$ of recombinant microtubules display minus-end dynamics under our assay conditions. Early studies reported faster polymerization rates for $\alpha \beta$ III-tubulin ( $\alpha$ denotes here an unknown mixture of $\alpha$-tubulin isoforms) immunopurified from brain tubulin preparations than for brain tubulin (15). Those studies also found that $\alpha \beta I I I-t u b u l i n$ immunopurified from brain tubulin preparations 


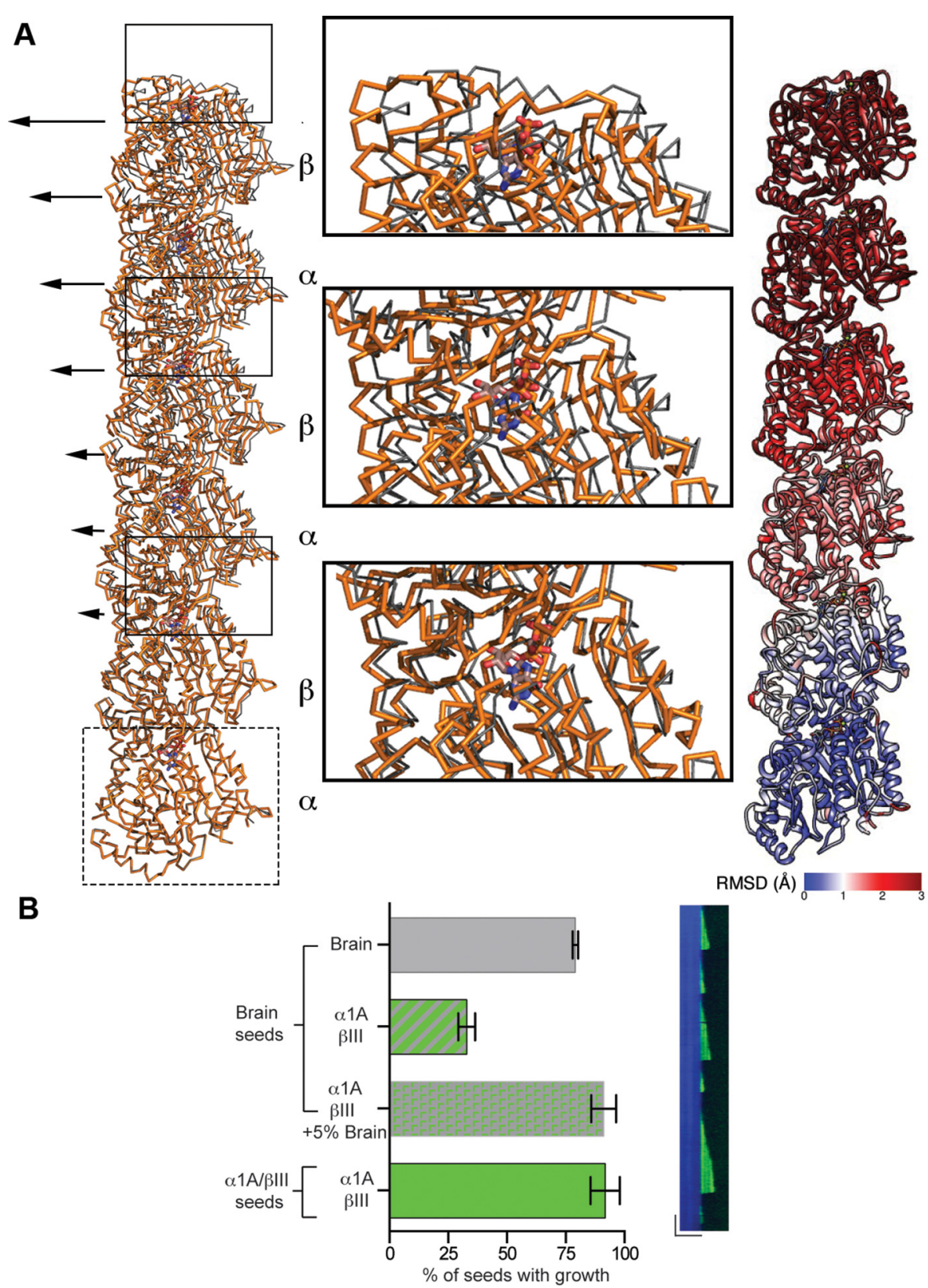

FIGURE 3. Comparison between $\alpha \mathbf{A} / \boldsymbol{\beta}$ III and mosaic brain 14 protofilament microtubule structures. $A$, left panel, dimer displacement compared with the structure of mosaic brain microtubules PDB 3JAT (28) as viewed from the microtubule lumen. The boxed $\alpha 1 \mathrm{~A}$-tubulin protomer from the $\alpha 1 \mathrm{~A} / \beta \mathrm{III}$ structure (orange $C \alpha$ trace) was superimposed on the $\alpha$-tubulin protomer from the brain microtubule structure ( $g r a y C \alpha$ trace). Arrows indicate the gradual increase in displacement of the $\alpha 1 \mathrm{~A} / \beta$ III heterodimers as one advances toward the plus-end of the protofilament. The GTP and GMPCPP in the N-site of $\alpha$-tubulin and the E-site of $\beta$-tubulin are shown as ball-and-stick. Middle panel, zoomed in view of regions highlighted by boxes in the left panel showing details of the displacement between the dimers from the recombinant $\alpha 1 \mathrm{~A} / \beta \mathrm{III}$ and brain microtubule structures; Right panel, three $\alpha 1 \mathrm{~A} / \beta \mathrm{ll}$ heterodimers within one protofilament colored according to main chain displacement from the brain microtubule structure. $B$, left panel, percentage of seeds that nucleate microtubules at $6 \mu \mathrm{m}$ tubulin. Brain, $\alpha 1 \mathrm{~A} / \beta \mathrm{III}, \alpha 1 \mathrm{~A} / \beta \mathrm{III}+5 \%$ brain tubulin elongated from brain seeds and $\alpha 1 \mathrm{~A} / \beta \mathrm{II}$-tubulin elongated from $\alpha 1 \mathrm{~A} / \beta \mathrm{III}$ seeds. More than 100 seeds across multiple chambers were counted for these measurements. Right panel, kymograph of microtubule growth for recombinant $\alpha 1 \mathrm{~A} / \beta \mathrm{Ill}$ at $5.7 \mu \mathrm{m}$ supplemented with $5 \%$ Hilyte 488 brain tubulin ( $0.3 \mu \mathrm{M}$ ) from brain GMPCPP seeds showing incorporation of the brain tubulin into the $\alpha 1 \mathrm{~A} / \beta$ III lattice. Horizontal and vertical scale bar, $5 \mu \mathrm{m}$ and 2 min, respectively.

had higher dynamicity than brain tubulin, although our measurements with recombinant $\alpha 1 \mathrm{~A} / \beta \mathrm{III}$ show lower dynamicity for this species than for brain microtubules $(1.31 \pm 0.05$ $\mu \mathrm{m} / \mathrm{min}$ versus $2.30 \pm 0.07 \mu \mathrm{m} / \mathrm{min}$ for $\alpha 1 \mathrm{~A} / \beta \mathrm{III}$ and brain, respectively; "Experimental Procedures"). However, it is important to note that the tubulin used in these earlier studies had an unknown $\alpha$-tubulin composition and a poorly defined mixture of diverse post-translational modifications, unlike our recombinant tubulin, which contains a single $\alpha$ - and $\beta$-tubulin isoform and is unmodified (Fig. $1 A$ and "Experimental Proce- dures"). It is unclear at this point whether the subtle differences in dynamics we observe between the recombinant $\alpha 1 \mathrm{~A} / \beta \mathrm{III}$ microtubules and heterogeneous mosaic brain microtubules are due to isoform differences, purification method, and/or the abundant and diverse post-translational modifications found on brain microtubules. Future studies with recombinantly expressed isoforms and quantitatively defined post-translationally modified tubulin using the expression and purification system described here will shed light on their individual contributions to dynamic instability parameters. 

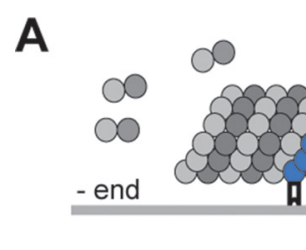

Biotinylated

GMPCPP seed

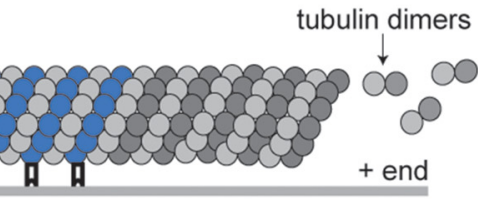

NeutrAvidin coated glass
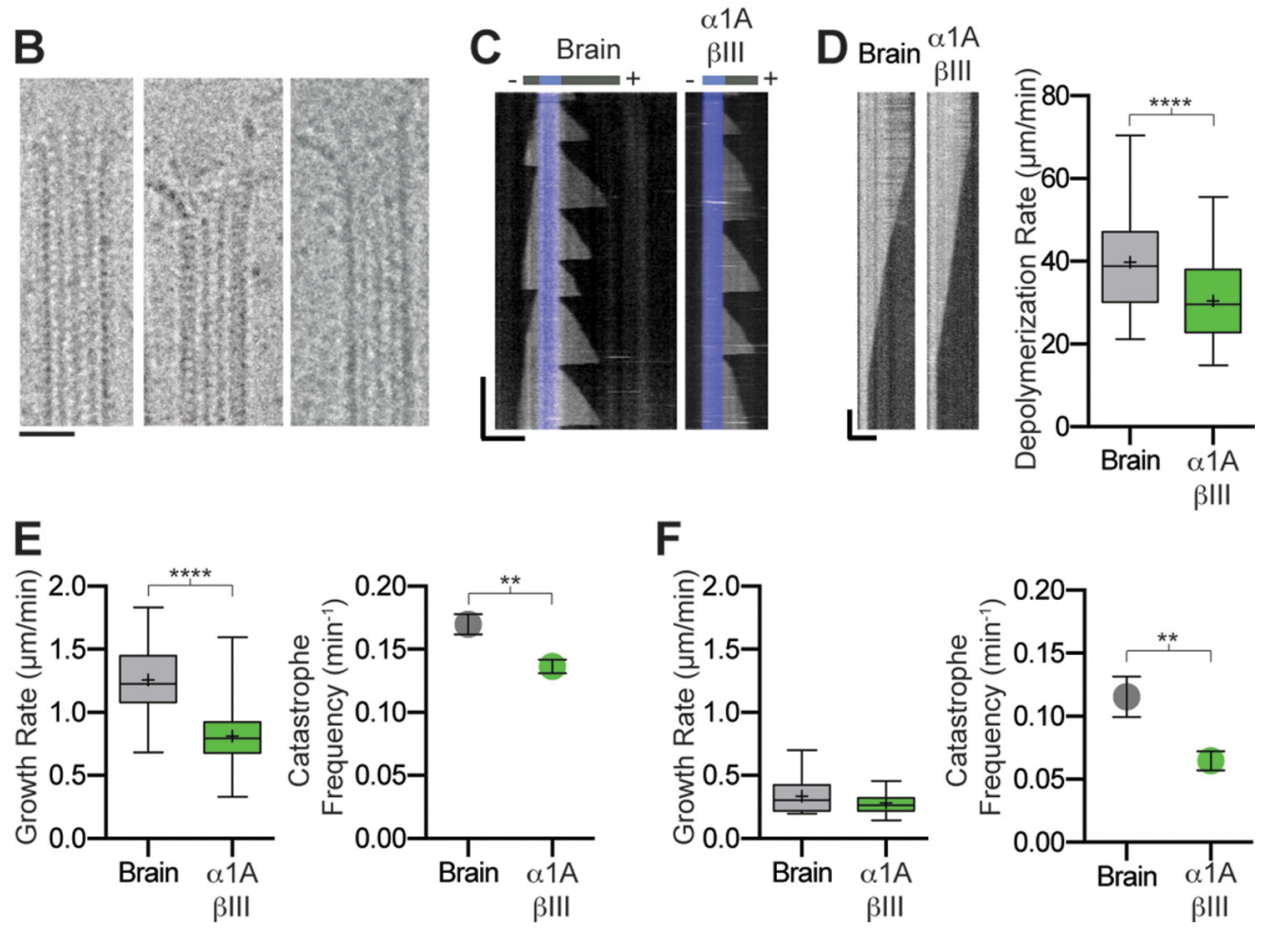

FIGURE 4. Dynamic parameters of recombinant human $\boldsymbol{\alpha} \mathbf{1} \mathbf{A} / \boldsymbol{\beta}$ III microtubules. $A$, schematic of assay design (see under "Experimental Procedures"). $B$, micrographs of representative dynamic $\alpha 1 \mathrm{~A} / \beta \mathrm{III}$ microtubule ends. Scale bar, $20 \mathrm{~nm}$. C, kymographs showing typical microtubule growth for brain and recombinant $\alpha 1 \mathrm{~A} / \beta \mathrm{lll}$-tubulin at $9 \mu \mathrm{m}$. Blue marks the GMPCPP seed. Horizontal and vertical scale bars, $5 \mu \mathrm{m}$ and 5 min, respectively. D, left panel, kymographs showing a typical depolymerization event for brain and $\alpha 1 \mathrm{~A} / \beta \mathrm{III}$ microtubules. Horizontal and vertical scale bar, $5 \mu \mathrm{m}$ and $2 \mathrm{~s}$, respectively. Right panel, Tukey plot showing plus-end depolymerization rates at $9 \mu \mathrm{m}$ tubulin; $n=55$ and 58 events for brain and $\alpha 1 \mathrm{~A} / \beta \mathrm{III}$ microtubules, respectively. E, plus-end dynamics of brain and $\alpha 1 \mathrm{~A} / \beta \mathrm{III}$-tubulin at $9 \mu \mathrm{m}$ tubulin. Left panel, box-whisker plot (whiskers indicate minimum and maximum) showing growth rates; $n=255$ and 504 events for brain and $\alpha 1 \mathrm{~A} / \beta \mathrm{III}$-tubulin, respectively. Right panel, catastrophe frequencies; $n=48$ and 167 microtubules for brain and $\alpha 1 \mathrm{~A} / \beta \mathrm{III}$-tubulin, respectively. F, minus-end dynamics of brain and $\alpha 1 \mathrm{~A} / \beta$ III-tubulin at $9 \mu \mathrm{m}$ tubulin. Left panel, box-whisker plot (whiskers indicate minimum and maximum) showing growth rates; $n=32$ and 25 events for brain and $\alpha 1 \mathrm{~A} / \beta$ III-tubulin, respectively. Right panel, catastrophe frequencies; $n=7$ and 16 microtubules for brain and $\alpha 1 \mathrm{~A} / \beta \mathrm{III}$-tubulin, respectively. Error bars represent S.E. ${ }^{* *}$ and ${ }^{* * *}, p$ values $<0.01$ and $<0.0001$, respectively determined by unpaired $t$ test.

\section{Discussion}

Using our dual-tag purification system for recombinant tubulin, we report for the first time the structure and in vitro dynamics parameters for isotypically pure human unmodified microtubules, an essential and important initial step in quantitatively establishing the correlates between sequence and dynamics for mammalian microtubules. The dual-tag selection system is necessary as a single-tag purification strategy results in significant levels of contamination with endogenous tubulin ( $~ 30 \%$ of insect $\alpha$-tubulin if $\alpha$-tubulin is not selected via an affinity tag). Thus, our tagging and purification strategy allows the characterization of both $\alpha$ - and $\beta$-tubulin engineered constructs. The majority of in vitro dynamics studies presently performed use heterogeneous mosaic brain microtubules with isoform composition and post-translational modifications different from those found in vivo, for example in an epithelial cell or the axonal or dendritic compartment of a neuron. A recent study revealed different activities of the Saccharomyces cerevisiae Stu2p on yeast microtubules compared with heterogeneous brain microtubules (50), indicating the importance of examining the effects of regulators with the physiologically relevant tubulin substrate. Our study establishes a system to examine the dynamics of mammalian microtubules with well defined tubulin species and opens the way to study tubulin isoform-specific effects of microtubule-associated proteins and motors and to uncover the tubulin sequence elements critical for their recruitment and activation.

Author Contributions-A. R.-M. conceived the project. A. V. and J. O.S. performed and analyzed the dynamics assays. J. A. determined EM structure, and A.S. purified recombinant tubulin. All authors interpreted data. A. R.-M. wrote the manuscript with contributions from A. V., J. O. S., J. A., and C. A. M.

\section{References}

1. Mitchison, T., and Kirschner, M. (1984) Dynamic instability of microtubule growth. Nature 312, 237-242

2. Bieling, P., Laan, L., Schek, H., Munteanu, E. L., Sandblad, L., Dogterom, M., Brunner, D., and Surrey, T. (2007) Reconstitution of a microtubule plus-end tracking system in vitro. Nature 450, 1100-1105 
3. Brouhard, G. J., Stear, J. H., Noetzel, T. L., Al-Bassam, J., Kinoshita, K., Harrison, S. C., Howard, J., and Hyman, A. A. (2008) XMAP215 is a processive microtubule polymerase. Cell 132, $79-88$

4. Leandro-García, L. J., Leskelä, S., Landa, I., Montero-Conde, C., LópezJiménez, E., Letón, R., Cascón, A., Robledo, M., and Rodríguez-Antona, C. (2010) Tumoral and tissue-specific expression of the major human $\beta$-tubulin isotypes. Cytoskeleton 67, 214-223

5. Miller, K. E., and Joshi, H. C. (1996) Tubulin transport in neurons. J. Cell Biol. 133, 1355-1366

6. Yu, I., Garnham, C. P., and Roll-Mecak, A. (2015) Writing and reading the tubulin code. J. Biol. Chem. 290, 17163-17172

7. Verhey, K. J., and Gaertig, J. (2007) The tubulin code. Cell Cycle 6, $2152-2160$

8. Weisenberg, R. C. (1972) Microtubule formation in vitro in solutions containing low calcium concentrations. Science 177, 1104-1105

9. Sullivan, K. F., and Cleveland, D. W. (1986) Identification of conserved isotype-defining variable region sequences for four vertebrate beta tubulin polypeptide classes. Proc. Natl. Acad. Sci. U.S.A. 83, 4327-4331

10. Banerjee, A., Roach, M. C., Wall, K. A., Lopata, M. A., Cleveland, D. W., and Ludueña, R. F. (1988) A monoclonal antibody against the type II isotype of $\beta$-tubulin. Preparation of isotypically altered tubulin. J. Biol. Chem. 263, 3029-3034

11. Garnham, C. P., and Roll-Mecak, A. (2012) The chemical complexity of cellular microtubules: tubulin post-translational modification enzymes and their roles in tuning microtubule functions. Cytoskeleton 69, 442-463

12. Zambito, A. M., Knipling, L., and Wolff, J. (2002) Charge variants of tubulin, tubulin S, membrane-bound and palmitoylated tubulin from brain and pheochromocytoma cells. Biochim. Biophys. Acta 1601, 200-207

13. Trinczek, B., Marx, A., Mandelkow, E. M., Murphy, D. B., and Mandelkow, E. (1993) Dynamics of microtubules from erythrocyte marginal bands. Mol. Biol. Cell 4, 323-335

14. Newton, C. N., DeLuca, J. G., Himes, R. H., Miller, H. P., Jordan, M. A., and Wilson, L. (2002) Intrinsically slow dynamic instability of HeLa cell microtubules in vitro. J. Biol. Chem. 277, 42456-42462

15. Panda, D., Miller, H. P., Banerjee, A., Ludueña, R. F., and Wilson, L. (1994) Microtubule dynamics in vitro are regulated by the tubulin isotype composition. Proc. Natl. Acad. Sci. 91, 11358-11362

16. Minoura, I., Hachikubo, Y., Yamakita, Y., Takazaki, H., Ayukawa, R., Uchimura, S., and Muto, E. (2013) Overexpression, purification, and functional analysis of recombinant human tubulin dimer. FEBS Lett. 587, $3450-3455$

17. Valenstein, M. L., and Roll-Mecak, A. (2016) Graded control of microtubule severing by tubulin glutamylation. Cell 164, 911-921

18. Sirajuddin, M., Rice, L. M., and Vale, R. D. (2014) Regulation of microtubule motors by tubulin isotypes and post-translational modifications. Nat. Cell Biol. 16, 335-344

19. Atherton, J., Farabella, I., Yu, I. M., Rosenfeld, S. S., Houdusse, A., Topf, M., and Moores, C. A. (2014) Conserved mechanisms of microtubulestimulated ADP release, ATP binding, and force generation in transport kinesins. Elife 3, e03680

20. Kremer, J. R., Mastronarde, D. N., and McIntosh, J. R. (1996) Computer visualization of three-dimensional image data using IMOD. J. Struct. Biol. 116, 71-76

21. Abrishami, V., Vargas, J., Li, X., Cheng, Y., Marabini, R., Sorzano, C. Ó., and Carazo, J. M. (2015) Alignment of direct detection device micrographs using a robust Optical Flow approach. J. Struct. Biol. 189, 163-176

22. de la Rosa-Trevín, J. M., Otón, J., Marabini, R., Zaldívar, A., Vargas, J., Carazo, J. M., and Sorzano, C. O. (2013) Xmipp 3.0: an improved software suite for image processing in electron microscopy. J. Struct. Biol. 184, 321-328

23. Mindell, J. A., and Grigorieff, N. (2003) Accurate determination of local defocus and specimen tilt in electron microscopy. J. Struct. Biol. 142, 334-347

24. Ludtke, S. J., Baldwin, P. R., and Chiu, W. (1999) EMAN: semiautomated software for high-resolution single-particle reconstructions. J. Struct. Biol. 128, 82-97

25. Sindelar, C. V., and Downing, K. H. (2007) The beginning of kinesin's forcegenerating cycle visualized at 9-A resolution. J. Cell Biol. 177, 377-385
26. Chen, S., McMullan, G., Faruqi, A. R., Murshudov, G. N., Short, J. M., Scheres, S. H., and Henderson, R. (2013) High-resolution noise substitution to measure overfitting and validate resolution in $3 \mathrm{D}$ structure determination by single particle electron cryomicroscopy. Ultramicroscopy 135, 24-35

27. Emsley, P., Lohkamp, B., Scott, W. G., and Cowtan, K. (2010) Features and development of Coot. Acta Crystallogr. D Biol. Crystallogr. 66, 486-501

28. Zhang, R., Alushin, G. M., Brown, A., and Nogales, E. (2015) Mechanistic origin of microtubule dynamic instability and its modulation by EB proteins. Cell 162, 849-859

29. Brown, A., Long, F., Nicholls, R. A., Toots, J., Emsley, P., and Murshudov, G. (2015) Tools for macromolecular model building and refinement into electron cryo-microscopy reconstructions. Acta Crystallogr. D Biol. Crystallogr. 71, 136-153

30. Pecqueur, L., Duellberg, C., Dreier, B., Jiang, Q., Wang, C., Plückthun, A., Surrey, T., Gigant, B., and Knossow, M. (2012) A designed ankyrin repeat protein selected to bind to tubulin caps the microtubule plus end. Proc. Natl. Acad. Sci. U.S.A. 109, 12011-12016

31. Nicholls, R. A., Long, F., and Murshudov, G. N. (2012) Low-resolution refinement tools in REFMAC5. Acta Crystallogr. D Biol. Crystallogr. 68, $404-4.17$

32. Gell, C., Bormuth, V., Brouhard, G. J., Cohen, D. N., Diez, S., Friel, C. T., Helenius, J., Nitzsche, B., Petzold, H., Ribbe, J., Schäffer, E., Stear, J. H., Trushko, A., Varga, V., Widlund, P. O., Zanic, M., and Howard, J. (2010) Microtubule dynamics reconstituted in vitro and image by single-molecule fluorescence microscopy. Methods Cell Biol. 95, 221-245

33. Szyk, A., Deaconescu, A. M., Spector, J., Goodman, B., Valenstein, M. L., Ziolkowska, N. E., Kormendi, V., Grigorieff, N., and Roll-Mecak, A. (2014) Molecular basis for age-dependent microtubule acetylation by tubulin acetyltransferase. Cell 157, 1405-1415

34. Ziółkowska, N. E., and Roll-Mecak, A. (2013) in Adhesion Protein Protocols (Coutts, A. S., ed) pp. 323-334, Humana Press, New York

35. Toso, R. J., Jordan, M. A., Farrell, K. W., Matsumoto, B., and Wilson, L. (1993) Kinetic stabilization of microtubule dynamic instability in vitro by vinblastine. Biochemistry 32, 1285-1293

36. Burgoyne, R. D., Cambray-Deakin, M. A., Lewis, S. A., Sarkar, S., and Cowan, N. J. (1988) Differential distribution of $\beta$-tubulin isotypes in cerebellum. EMBO J. 7, 2311-2319

37. Kavallaris, M. (2010) Microtubules and resistance to tubulin-binding agents. Nat. Rev. Cancer 10, 194-204

38. Peris, L., Thery, M., Fauré, J., Saoudi, Y., Lafanechère, L., Chilton, J. K., Gordon-Weeks, P., Galjart, N., Bornens, M., Wordeman, L., Wehland, J., Andrieux, A., and Job, D. (2006) Tubulin tyrosination is a major factor affecting the recruitment of CAP-Gly proteins at microtubule plus ends. J. Cell Biol. 174, 839-849

39. Peris, L., Wagenbach, M., Lafanechère, L., Brocard, J., Moore, A. T., Kozielski, F., Job, D., Wordeman, L., and Andrieux, A. (2009) Motor-dependent microtubule disassembly driven by tubulin tyrosination. J. Cell Biol. 185, 1159-1166

40. Vale, R. D., Coppin, C. M., Malik, F., Kull, F. J., and Milligan, R. A. (1994) Tubulin GTP hydrolysis influences the structure, mechanical properties, and kinesin-driven transport of microtubules. J. Biol. Chem. 269, $23769-23775$

41. Nogales, E., Wolf, S. G., and Downing, K. H. (1998) Structure of the $\alpha \beta$ tubulin dimer by electron crystallography. Nature 391, 199-203

42. Garnham, C. P., Vemu, A., Wilson-Kubalek, E. M., Yu, I., Szyk, A., Lander, G. C., Milligan, R. A., and Roll-Mecak, A. (2015) Multivalent microtubule recognition by tubulin tyrosine ligase-like family glutamylases. Cell 161, $1112-1123$

43. Roll-Mecak, A. (2015) Intrinsically disordered tubulin tails: complex tuners of microtubule functions? Semin. Cell Dev. Biol. 37, 11-19

44. Alushin, G. M., Ramey, V. H., Pasqualato, S., Ball, D. A., Grigorieff, N., Musacchio, A., and Nogales, E. (2010) The Ndc80 kinetochore complex forms oligomeric arrays along microtubules. Nature 467, 805-810

45. Alushin, G. M., Lander, G. C., Kellogg, E. H., Zhang, R., Baker, D., and Nogales, E. (2014) High-resolution microtubule structures reveal the structural transitions in $\alpha \beta$-tubulin upon GTP hydrolysis. Cell 157, 1117-1129

46. Horio, T., and Hotani, H. (1986) Visualization of the dynamic instabil- 
ity of individual microtubules by dark-field microscopy. Nature 321, 605-607

47. Mandelkow, E. M., Mandelkow, E., and Milligan, R. A. (1991) Microtubule dynamics and microtubule caps: a time-resolved cryo-electron microscopy study. J. Cell Biol. 114, 977-991

48. Gardner, M. K., Charlebois, B. D., Jánosi, I. M., Howard, J., Hunt, A. J., and Odde, D. J. (2011) Rapid microtubule self-assembly kinetics. Cell 146, 582-592

49. Grishchuk, E. L., Molodtsov, M. I., Ataullakhanov, F. I., and McIntosh, J. R. (2005) Force production by disassembling microtubules. Nature 438, 384-388
50. Podolski, M., Mahamdeh, M., and Howard, J. (2014) Stu2, the budding yeast XMAP215/Dis1 homolog, promotes assembly of yeast microtubules by increasing growth rate and decreasing catastrophe frequency. J. Biol. Chem. 289, 28087-28093

51. Cardone, G., Heymann, J. B., and Steven, A. C. (2013) One number does not fit all: mapping local variations in resolution in cryo-EM reconstructions. J. Struct. Biol. 184, 226-236

52. Sousa, D., and Grigorieff, N. (2007) Ab initio resolution measurement for single particle structures. J. Struct. Biol. 157, 201-210 
Structure and Dynamics of Single-isoform Recombinant Neuronal Human Tubulin Annapurna Vemu, Joseph Atherton, Jeffrey O. Spector, Agnieszka Szyk, Carolyn A. Moores and Antonina Roll-Mecak

J. Biol. Chem. 2016, 291:12907-12915. doi: 10.1074/jbc.C116.731133 originally published online April 25, 2016

Access the most updated version of this article at doi: 10.1074/jbc.C116.731133

Alerts:

- When this article is cited

- When a correction for this article is posted

Click here to choose from all of JBC's e-mail alerts

Supplemental material:

http://www.jbc.org/content/suppl/2016/04/25/C116.731133.DC1

This article cites 51 references, 15 of which can be accessed free at http://www.jbc.org/content/291/25/12907.full.html\#ref-list-1 
Structure and dynamics of single-isoform recombinant neuronal human tubulin

Annapurna Vemu ${ }^{1 \S}$, Joseph Atherton ${ }^{2 \S}$, Jeffrey O. Spector ${ }^{1 \S}$, Agnieszka Szyk ${ }^{1}$, Carolyn A. Moores $^{2, *}$ and Antonina Roll-Mecak ${ }^{1,3, *}$ 
SUPPLEMENTAL TABLE 1. Refinement statistics and model geometry

Refinement statistics and model geometry from REFMAC v5.8 (29) and MolProbity (51).

\begin{tabular}{|l|l|}
\hline Resolution for refinement & $4 \AA$ \\
\hline Map sharpening B factor & -180 \\
\hline FSC $_{\text {average }}{ }^{2}$ & 0.79 \\
\hline Rms deviations (bonds) $\left(\AA^{2}\right)$ & 0.008 \\
\hline Rms deviations (angles) $\left(\AA^{2}\right)$ & 1.438 \\
\hline Molprobity Score & $1.75\left(100^{\text {th }}\right.$ percentile $)$ \\
\hline Clashscore, all atoms & $7.39(100$ th percentile $)$ \\
\hline Poor rotamers (\%) & $1.18 \%$ \\
\hline Ramachandran plot favoured $(\%)$ & $95.75 \%$ \\
\hline Ramachandran outliers $(\%)$ & $0.12 \%$ \\
\hline
\end{tabular}

${ }^{\mathrm{a}}$ aFSCaverage $=\Sigma\left(\mathrm{N}_{\text {shell }} \mathrm{FSC}_{\text {shell }}\right) / \Sigma\left(\mathrm{N}_{\text {shell }}\right)$, where $\mathrm{FSC}_{\text {shell }}$ is the FSC in a given shell, $\mathrm{N}_{\text {shell }}$ is the number of structural factors in the shell. $\left.\mathrm{FSC}_{\text {shell }}=\Sigma\left(\mathrm{F}_{\text {model }} \mathrm{F}_{\mathrm{EM}}\right) /\left(\sqrt{ } \Sigma\left(|\mathrm{F}| 2_{\text {model }}\right)\right) \sqrt{ } \Sigma\left(\mathrm{F}_{\mathrm{EM}}^{2}\right)\right)$ 


\section{SUPPLEMENTAL MOVIE 1}

Dynamics of recombinant human $\alpha 1 \mathrm{~A} / \beta$ III microtubules imaged by darkfield microscopy. Dynamic recombinant human $\alpha 1 \mathrm{~A} / \beta \mathrm{III}$ microtubules at $9 \mu \mathrm{M}$ tubulin imaged by darkfield microscopy. Blue marks the GMPCPP seed. Recorded at 12 frames per minute, played back at 30 frames per second.

\section{SUPPLEMENTAL MOVIE 2}

Dynamics of heterogeneous brain microtubules imaged by darkfield microscopy.

Dynamic brain microtubules at $9 \mu \mathrm{M}$ tubulin imaged by darkfield microscopy. Blue marks the GMPCPP seed. Recorded at 12 frames per minute, played back at 30 frames per second.

\section{SUPPLEMENTAL MOVIE 3}

Depolymerizing human $\alpha 1 \mathrm{~A} / \beta$ III microtubule imaged by darkfield microscopy at high temporal resolution. Scale bar, $2.5 \mu \mathrm{m}$. Recorded at 40 frames per second played back at 30 frames per second. 